\title{
Usefulness of Stress-Derived E/e' Ratio in Asymptomatic Hypertensive Patients
}

\author{
Ragab A. Mahfouz Mohammed Abdelhamed Islam Galal Moataz Elsanan \\ Department of Cardiology, Zagazig University Hospital, Zagazig, Egypt
}

\section{Keywords}

Diastolic blood pressure $\cdot$ Stress echo $\cdot$ Hypertension .

Microvascular function

\begin{abstract}
Purpose: We sought to investigate the usefulness of stress echocardiography-derived $\mathrm{E} / \mathrm{e}^{\prime}$ in predicting subclinical atherosclerosis in asymptomatic hypertensive patients. Materials and Methods: 71 newly diagnosed untreated hypertensive patients ( $48 \pm 13$ years, $65 \%$ males) and 30 age- and sexmatched healthy controls were recruited. Resting and exercise echocardiography was performed to assess resting diastolic blood pressure and the diastolic stress parameters. Coronary flow reserve (CFR) was evaluated as well. Results: Based on CFR values, newly diagnosed, untreated hypertensives were stratified into hypertensives with microvascular dysfunction (MVD; 34 patients had CFR $<2.0$ ) and those without MVD (37 patients had CFR 22.0 ). Patients with MVD had a significantly higher $C$-reactive protein level $(p<0.05)$ and lower metabolic equivalent values $(p<0.05)$. With resting echocardiography, only the left atrial volume index (LAVI) was significantly increased in those with MVD compared with those without MVD and controls $(p<0.05)$. With exercise echo, the E/e' was significantly increased in MVD patients compared with those without MVD and controls $(p<$ 0.001). Importantly, the percentage of subjects with exercise
\end{abstract}

$E / e^{\prime} \geq 15$ was $76.5 \%$ (26 patients in the group with MVD), $4.1 \%$ (3 patients in the group without MVD), and $0 \%$ in controls. At univariate analysis, high-sensitivity $C$-reactive protein $(p<0.05)$, LAVI $(p<0.05)$, and exercise E/e' $(p<0.001)$ were independently associated with reduced CFR. On the other hand, at multivariate analysis, only exercise E/e' was the independent predictor of reduced CFR in newly diagnosed hypertensives. Conclusion: We have demonstrated significant associations between exercise-derived raised left ventricular pressure and coronary MVD in newly diagnosed untreated hypertensive patients. Herein, we supposed that exercise-derived E/e' could predict subclinical atherosclerosis and might be a risk parameter for newly diagnosed untreated hypertensive patients.

(c) 2020 S. Karger AG, Basel

\section{Introduction}

The pathogenesis of impaired microvascular function in hypertensive patients without obstructive coronary arteries is still unclear. Left ventricular (LV) hypertrophy in patients with hypertension is a well-known contributor of impaired coronary vasodilator capacity $[1,2]$; nonetheless, impaired coronary reactivity may exist in some patients with hypertension in the absence of LV hypertrophy and in asymptomatic hypertensive subjects [3].

\footnotetext{
Ragab A. Mahfouz

Department of Cardiology, Zagazig University Hospital

10 Naeem Street, Alsharkeah

AlZohor Region, Zagazig (Egypt)

ragabaziza61@yahoo.commailto:
} 
Diastolic stress echocardiography is an important tool to detect latent diastolic dysfunction in a significant proportion of patients, who are classified as normal with resting echocardiography. Definitively diastolic stress echocardiography is considered abnormal when the septal E/e' ratio is $>15$, peak tricuspid regurgitant velocity is $>2.8 \mathrm{~m} / \mathrm{s}$ with exercise, and the septal e' velocity is $<8 \mathrm{~cm} / \mathrm{s}$ at rest [4-6].

D'Andrea et al. [7] have reported that LV diastolic function was impaired in patients with microvascular angina compared with asymptomatic controls. Furthermore, Nelson et al. [8] found that women with signs and symptoms of ischemia in the absence of obstructive coronary artery disease had significant diastolic dysfunction compared with age-matched control individuals.

We hypothesized that microvascular dysfunction (MVD) present in newly diagnosed untreated hypertensive patients could be associated with subclinical diastolic dysfunction (diastolic heart failure). Therefore, the purpose of our research was to study the relation between exercise LV filling pressure as estimated by exercise-derived E/e' and coronary flow reserve (CFR) in newly diagnosed untreated hypertensive patients.

\section{Study Subjects and Methods}

In a prospective study, 71 newly diagnosed hypertensive patients (age: $48 \pm 13$ years, $65 \%$ males) who visited the hypertensive clinic in the Cardiology Department, Zagazig University Hospital, and 30 age- and gender-matched healthy control subjects were enrolled for a prospective study. Essential hypertension was diagnosed based on the definition of seated systolic blood pressure $>140 \mathrm{~mm} \mathrm{Hg}$ and/or diastolic blood pressure $>90 \mathrm{~mm} \mathrm{Hg}$ during at least 3 measurements and $>125 / 80 \mathrm{~mm} \mathrm{Hg}$ during 24-h ambulatory monitoring [9]. Patients were included if they met the following inclusion criteria: untreated hypertensive patients, no symptoms or history of other cardiovascular disease, LV ejection fraction $\geq 50 \%$, absence of regional LV wall motion abnormalities with echocardiographic assessment. Exclusion criteria were: atrial fibrillation, history of angina or ischemic heart disease, congenital heart disease, significant valvular disease, malignancy, chronic obstructive pulmonary disease, obstructive sleep and patients with serum creatinine $>2.0 \mathrm{mg} / \mathrm{dL}$, and those with diabetes mellitus. Also, patients with a positive exercise stress test were excluded from the study because of the suspicion of significant coronary artery stenosis. Patients with newly diagnosed untreated hypertension were stratified based on the results of CFR values into 2 groups: hypertensive patients with MVD, which included 34 patients with CFR $<2.0$, and hypertensives without MVD, which included 37 patients with CFR $\geq 2.0$. All participants were informed of the study design, and written informed consent was obtained from every participant. Moreover, the study protocol was approved by the local hospital scientific and ethical committee.

Diastolic Stress Echo, Hypertension
Blood Pressure Measurements

The average of 3 sequential measurements was taken with 2 min apart after resting in a supine position for $10 \mathrm{~min}$ in a quiet room. Measurements were obtained using a suitable cuff of a mercury sphygmomanometer on the right upper arm.

\section{Rest and Treadmill Stress Echocardiography}

Echocardiography was performed using a commercially available ultrasound system (GE Vingmed Vivid 7, GE Medical, Milwaukee, WI, USA) with a $2.5-\mathrm{MHz}$ transducer. The resting echocardiographic parameters included LV end-systolic diameter and end-diastolic diameter, LV ejection fraction, LV mass index (g/ $\mathrm{m}^{2}$ ), and left atrial volume index (LAVI, $\mathrm{mL} / \mathrm{m}^{2}$ ) [10].

At the apical 4-chamber view, a 2-mm pulsed Doppler sample volume was placed at the tip of mitral valve leaflets to obtain the average of early (E) and late (A) mitral flow velocities by averaging of 3 cardiac cycles. Subsequently, the tissue Doppler modality was activated, and the filter was set with an adjusted Nyquist limit range of $15-20 \mathrm{~cm} / \mathrm{s}$. The gain and sample volume were minimized to allow for a clear tissue signal with minimal background noise. Then, the mitral annular velocity was measured by Doppler tissue imaging. With a sample volume of $2-5 \mathrm{~mm}$ the mean of the early diastolic (e') velocity of the septal and lateral mitral annulus was calculated. The resting E/e' was calculated.

All participants underwent a maximum symptom-limited treadmill exercise test as previously described [11], by using the standard Bruce protocol or modified Bruce protocol (Fukuda Denshi Co. Ltd., Tokyo, Japan). Two-dimensional echocardiographic images using a standard apical 4-chamber view, apical 2 -chamber view, and apical long-axis view were acquired at rest and immediately after peak exercise within $1 \mathrm{~min}$ after the termination of exercise and stored digitally. Exercise mitral inflow $\mathrm{E}$ and a velocities were measured. The early mitral annular (e') velocity was also measured. Then the exercise-derived $\mathrm{E} / \mathrm{e}$ ' ratio was calculated [12]. Increased LV filling pressure after exercise was defined as an $\mathrm{E} / \mathrm{e}^{\prime}$ ratio $\geq 15$ [13].

The correlations for interobserver and intraobserver variability for resting E/e' were 0.97 and 0.99 , respectively ( $p<0.001)$. Also, the correlations for interobserver and intraobserver variability for exercise E/e' were 0.93 and 0.95 ( $p<0.001)$, respectively.

\section{Assessment of CFR}

Noninvasive transthoracic echocardiographic assessment of CFR was performed using GE Vingmed Vivid 7 (GE Medical, Milwaukee, WI, USA), which was equipped with a 5-MHz phased array probe. Coronary flow velocity profiles in the left anterior descending artery were obtained using color-guided pulse wave Doppler sonography. The frame rate and flow velocity cutoff were minimized to optimize color flow imaging. The peak diastolic coronary flow velocity was measured at baseline and after adenosine infusion $(140 \mu \mathrm{g} / \mathrm{kg} / \mathrm{min})$ for $3 \mathrm{~min}$, as previously described [14]. The average of 3 cycles was obtained. CFR was calculated as the ratio of peak diastolic coronary velocity to resting peak diastolic coronary flow velocity. A CFR value of $\geq 2.0$ was accepted as normal [14].

Inter- and intraobserver variability for CFR was assessed in 20 subjects, and it was 4 and 3\%, respectively. 
Table 1. Coronary flow velocity parameters of all participants

\begin{tabular}{|c|c|c|c|}
\hline $\begin{array}{l}\text { Coronary flow } \\
\text { parameters }\end{array}$ & $\begin{array}{l}\text { Control } \\
(n=30)\end{array}$ & $\begin{array}{l}\text { No MVD } \\
(n=37)\end{array}$ & $\begin{array}{l}\text { MVD } \\
(n=34)\end{array}$ \\
\hline seline DPFV $\mathrm{cm}$ & $23.7 \pm 4.5$ & $24.7 \pm 5.0$ & $25.7 \pm 4.9$ \\
\hline Hyperemic DPFV, $\mathrm{cm} / \mathrm{s}$ & $76.4 \pm 15.2$ & $65.1 \pm 13.5$ & $41.5 \pm 11.4^{* *}$ \\
\hline Coronary flow reserve & $2.89 \pm 0.52$ & $2.75 \pm 0.49$ & $1.65 \pm 0.45^{* *}$ \\
\hline
\end{tabular}

** $p<0.001$ : comparison between hypertension with MVD versus each hypertension without MVD and controls. MVD, microvascular dysfunction; DPFV, diastolic peak flow velocity.

\section{Statistical Analysis}

Continuous variables are presented as the mean values $\pm \mathrm{SD}$, while categorical variables were presented as numbers or percentages as appropriate. Analysis of variance and post hoc analysis tests were made for group differences. Pearson's correlation was used to determine correlations between continuous variables. Univariate and multivariate analysis was used to estimate the predictors of reduced CFR. Statistical analysis was performed using standard statistical software (SPSS version 11.5; Chicago, USA).

\section{Results}

Of 71 newly diagnosed hypertensive patients, 34 had CFR $<2.0$ (group with MVD) and 37 patients with CFR $\geq 2.0$ (Table 1). Table 2 presents the demographic characteristics, laboratory data and treadmill parameters of all participants. Both hypertensive patients with and without MVD had higher systolic blood pressure, diastolic blood pressure, and high-sensitivity C-reactive protein than controls. The results showed that CFR was significantly decreased $(p<0.001)$ in hypertensive patients with MVD compared with those without MVD and controls. Furthermore, metabolic equivalents (METs) were significantly lower $(<0.05)$ in hypertensive patients with MVD compared with those without MVD and controls.

As seen in Table 3, the results showed that LAVI $(p<$ $0.05)$ and LV mass index $(p<0.05)$ were significantly increased in hypertensive patients with MVD compared with hypertensive patients without MVD and controls, whereas other resting parameters were comparable among the 3 groups. With respect to exercise parameters, the current study shows that the exercise-derived E/e' ratio was significantly increased $(p<0.001)$ in patients with MVD compared with hypertensives without MVD and controls (Fig. 1). Interestingly, $76.5 \%$ of patients with MVD had increased E/e' $\geq 15$ versus only $4.1 \%$ in hypertensive patients without MVD $(p<0.001)$. Furthermore,

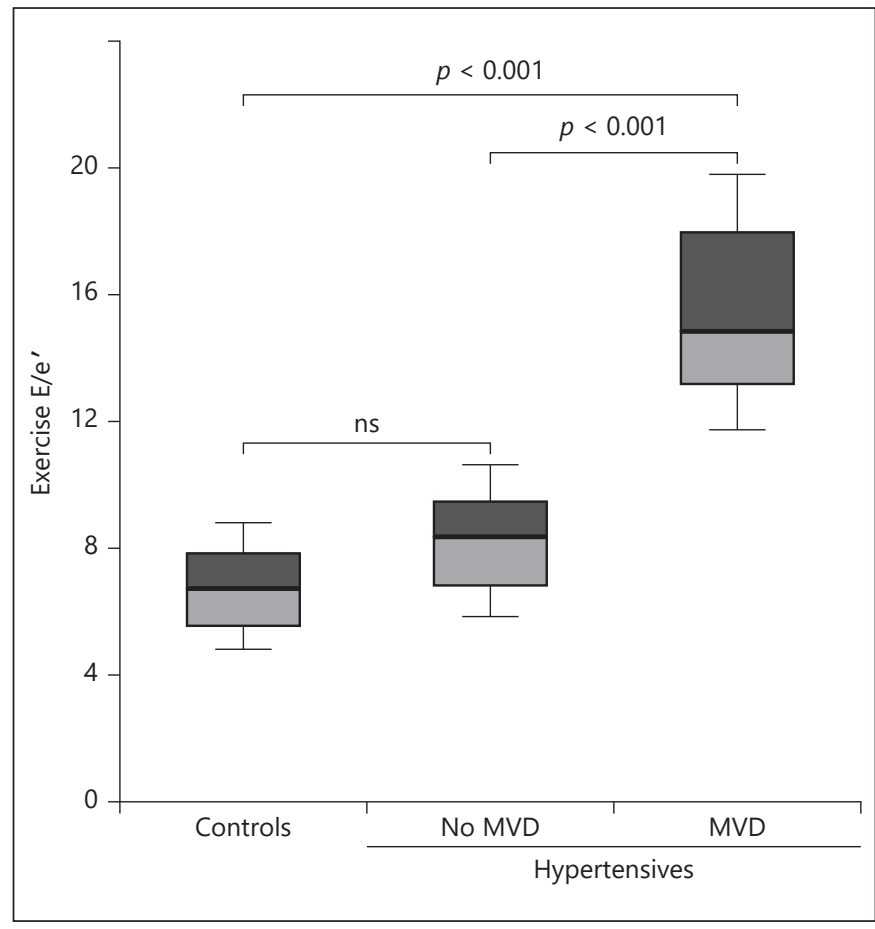

Fig. 1. Exercise E/e' ratio in hypertensives with microvascular dysfunction (MVD) compared with hypertensives without MVD and controls.

patients with rising E/e' had significantly lower MET values $(p<0.01)$, exercise duration $(p<0.05)$, and significantly lower CFR ( $p<0.001$; Table 4$)$.

Figure 2 shows that CFR was significantly correlated with exercise-derived E/e' $(r=-0.45, p<0.001)$.

Table 5 depicts the univariate and multivariate predictors of MVD in our cohort. At univariate analysis, highsensitivity C-reactive protein $(p<0.05)$, LAVI $(p<0.05)$, and resting exercise $\mathrm{E} / \mathrm{e}^{\prime}(p<0.001)$ were detected to be independent predictors of MVD (reduced CFR). On the other hand, at multivariate analysis, postexercise E/e' was the only independent predictor for MVD in asymptomatic hypertensive patients $(p<0.001)$.

\section{Discussion}

In our study we have demonstrated that reduced CFR was detected in 34 (48\%) of newly diagnosed untreated hypertensive patients. Furthermore, we found that raising LV filling pressure with exercise as evaluated with an increased exercise-derived E/e' ratio was the most powerful independent predictor of impaired coronary micro- 
Table 2. Baseline characteristics of all participants

\begin{tabular}{lccc}
\hline & $\begin{array}{c}\text { Control } \\
(n=30)\end{array}$ & $\begin{array}{c}\text { No MVD } \\
(n=37)\end{array}$ & $\begin{array}{c}\text { MVD } \\
(n=34)\end{array}$ \\
\hline Age, years & $49 \pm 6.5$ & $47.5 \pm 7.1$ & $48.6 \pm 7.5$ \\
Males, $n$ (\%) & $20(66.6)$ & $23(62.2)$ & $24(70.6)$ \\
Body mass index & $23.5 \pm 2.4$ & $25.1 \pm 2.1$ & $24.5 \pm 2.2$ \\
Current smoker, $n$ (\%) & $7(23.3)$ & $9(24.3)$ & $9(26.4)$ \\
Systolic blood pressure, mm Hg & $117.5 \pm 5.2$ & $149 \pm 7.1$ & $153.3 \pm 6.9^{*}$ \\
Diastolic blood pressure, mm Hg & $72.5 \pm 4.2$ & $89 \pm 5$ & $91.5 \pm 4.7^{*}$ \\
Diabetes, $n$ \%) & - & $5(13.5)$ & $4(11.8)$ \\
Total cholesterol, mg/dL & $180 \pm 35$ & $192 \pm 47$ & $199 \pm 38$ \\
Triglycerides, mg/dL & $109 \pm 43$ & $153 \pm 55$ & $149 \pm 48$ \\
LDL cholesterol, mg/dL & $115 \pm 21$ & $129 \pm 38$ & $135 \pm 34$ \\
HDL cholesterol, mg/dL & $47 \pm 6$ & $45 \pm 6$ & $44 \pm 7$ \\
Creatinine, mg/dL & $0.8 \pm 0.3$ & $1.0 \pm 0.3$ & $0.9 \pm 0.4$ \\
hs-CRP, mg/L & $2.1 \pm 0.9$ & $2.2 \pm 1.2$ & $4.2 \pm 1.5^{*}$ \\
Treadmill parameters & & & $85 \pm 13$ \\
$\quad$ HR rest & $81 \pm 9$ & $83 \pm 11$ & $149.4 \pm 20^{*}$ \\
$\quad$ HR exercise & $169 \pm 22$ & $164 \pm 18$ & $7.1 \pm 2.7^{*}$ \\
METs & $10.3 \pm 1.8$ & $9.9 \pm 1.7$ & \\
\hline
\end{tabular}

${ }^{*} p<0.05$ : comparison between hypertension with MVD versus each hypertension without MVD and controls. MVD, microvascular dysfunction; LDL, low-density lipoprotein; HDL, high-density lipoprotein; hs-CRP, highsensitivity C-reactive protein; HR, heart rate; METs, metabolic equivalents.

Table 3. Echocardiographic characteristics of all participants

\begin{tabular}{|c|c|c|c|}
\hline & Controls & $\begin{array}{l}\text { HTN without } \\
\text { MVD }(n=37)\end{array}$ & $\begin{array}{l}\text { HTN with } \\
\operatorname{MVD}(n=34)\end{array}$ \\
\hline IVSd, cm & $0.97 \pm 0.08$ & $1.05 \pm 0.09$ & $1.12 \pm 0.12^{*}$ \\
\hline PLVWd, cm & $1.00 \pm 0.07$ & $1.03 \pm 0.08$ & $1.08 \pm 0.12$ \\
\hline LVEDV index, $\mathrm{mL} / \mathrm{m}^{2}$ & $51.8 \pm 8.1$ & $52.5 \pm 9.3$ & $52.9 \pm 8.5$ \\
\hline LVESV index, $\mathrm{mL} / \mathrm{m}^{2}$ & $17.2 \pm 3.8$ & $18.1 \pm 4.1$ & $19.3 \pm 4.3$ \\
\hline $\mathrm{LAD}, \mathrm{cm}$ & $2.91 \pm 0.49$ & $3.11 \pm 0.37$ & $3.85 \pm 0.36^{*}$ \\
\hline LAVI, $\mathrm{mL} / \mathrm{m}^{2}$ & $23.7 \pm 2.9$ & $27.5 \pm 3.1$ & $39.3 \pm 2.8^{*}$ \\
\hline $\mathrm{EF}, \%$ & $66.9 \pm 2.8$ & $65.7 \pm 2.5$ & $63.5 \pm 2.3$ \\
\hline LVMI, g/m & $74.5 \pm 11.6$ & $79.3 \pm 13.4$ & $103.6 \pm 12.9^{*}$ \\
\hline \multicolumn{4}{|l|}{ Exercise echocardiography } \\
\hline \multicolumn{4}{|l|}{ LVEF, \% } \\
\hline Rest & $65.1 \pm 4.9$ & $64.8 \pm 5.0$ & $62.9 \pm 4.2$ \\
\hline Exercise & $71.5 \pm 7.4$ & $68.3 \pm 6.7$ & $66.5 \pm 6.6$ \\
\hline$\Delta$ & $5.9 \pm 3.5$ & $4.6 \pm 2.9$ & $4.2 \pm 2.5$ \\
\hline \multicolumn{4}{|l|}{$\mathrm{E} / \mathrm{A}$ ratio } \\
\hline Rest & $0.98 \pm 0.30$ & $0.96 \pm 0.22$ & $0.92 \pm 0.42$ \\
\hline Exercise & $1.09 \pm 0.38$ & $1.05 \pm 0.31$ & $0.95 \pm 0.45$ \\
\hline$\Delta$ & $0.09 \pm 0.49$ & $0.08 \pm 0.35$ & $0.04 \pm 0.53$ \\
\hline \multicolumn{4}{|l|}{ E/e' ratio } \\
\hline Rest & $5.5 \pm 1.9$ & $7.2 \pm 2.1$ & $8.9 \pm 3.1$ \\
\hline Exercise & $6.3 \pm 2.0$ & $7.7 \pm 2.4$ & $14.7 \pm 4.3^{* *}$ \\
\hline$\Delta$ & $0.3 \pm 2.6$ & $0.3 \pm 2.5$ & $4.1 \pm 3.5^{* *}$ \\
\hline Percent of $E / e^{\prime}$ ratio $\geq 15$ & 0 & $3(4.1)$ & $26(76.5)^{* *}$ \\
\hline
\end{tabular}

${ }^{*} p<0.05,{ }^{* *} p<0.001$ : comparison between hypertension (HTN) with MVD versus each HTN without MVD and controls. MVD, microvascular dysfunction; IVSd, diastolic interventricular septal thickness; PLVWd, diastolic posterior wall thickness; LVEDV, LVESV, LAD, left atrial dimension; LVMI; left ventricular mass index; LAVI, left atrial wall index; EF, ejection fraction; LVEF, left ventricular ejection fraction; $\Delta$, average change. 
Table 4. Treadmill parameters and coronary flow reserve of hypertensive patients as regards exercise E/e'

\begin{tabular}{llll}
\hline Variable & $\begin{array}{l}\text { No rising E/e' } \\
(n=42)\end{array}$ & $\begin{array}{l}\text { Rising E/e' } \\
(n=29)\end{array}$ & $\begin{array}{l}p \\
\text { value }\end{array}$ \\
\hline METs & $10.3 \pm 1.6$ & $6.90 \pm 1.5$ & $<0.01$ \\
Exercise duration, min & $9.50 \pm 0.5$ & $6.10 \pm 0.3$ & $<0.05$ \\
Coronary flow reserve & $2.91 \pm 0.53$ & $1.62 \pm 0.39$ & $<0.001$ \\
\hline
\end{tabular}

METs, metabolic equivalents.

vasculature in newly diagnosed untreated hypertensive patients.

With resting echocardiography, only LAVI was increased in hypertensives with reduced CFR; still, at multivariate analysis it is not a predictor of MVD. However, at multivariate analysis exercise-derived E/e' was significantly correlated with reduced CFR, and it was demonstrated to be a strong predictor for MVD. Moreover, patients with a rising exercise $\mathrm{E} / \mathrm{e}$ ' ratio had reduced exercise duration and METs compared with the nonrising E/e' group and controls.

In the resting condition, LV filling pressure is usually compensated; accordingly, it is difficult to identify the LV diastolic dysfunction without diastolic stress echo [15]. On the other hand, an early subclinical myocardial dysfunction might be masked by compensatory mechanisms, particularly at rest [16]. Holland et al. [16] found a significant association between raised exercise E/e' $>14.5$ and repeated hospitalization, independent and incremental to inducible ischemia [17].

Interestingly, the current study described that the newly diagnosed untreated hypertensive patients who exhibited raised exercise E/e' had impaired coronary MVD (reduced CFR). This finding may provide a clue for preclinical heart failure in newly diagnosed untreated hypertensive subjects and implies that coronary MVD might have a significant role in the pathogenesis of heart failure with preserved ejection fraction.

Another interesting issue is that the current study provided an important finding by showing that raising E/e' at exercise is correlated closely with reduced exercise tolerance as assessed by METs. This emphasizes that altered diastolic function during stress contributes to reduced exercise tolerance in newly diagnosed untreated hypertensive patients.

Ponikowski et al. [17] suggested that heart failure with preserved ejection fraction can be diagnosed on the basis

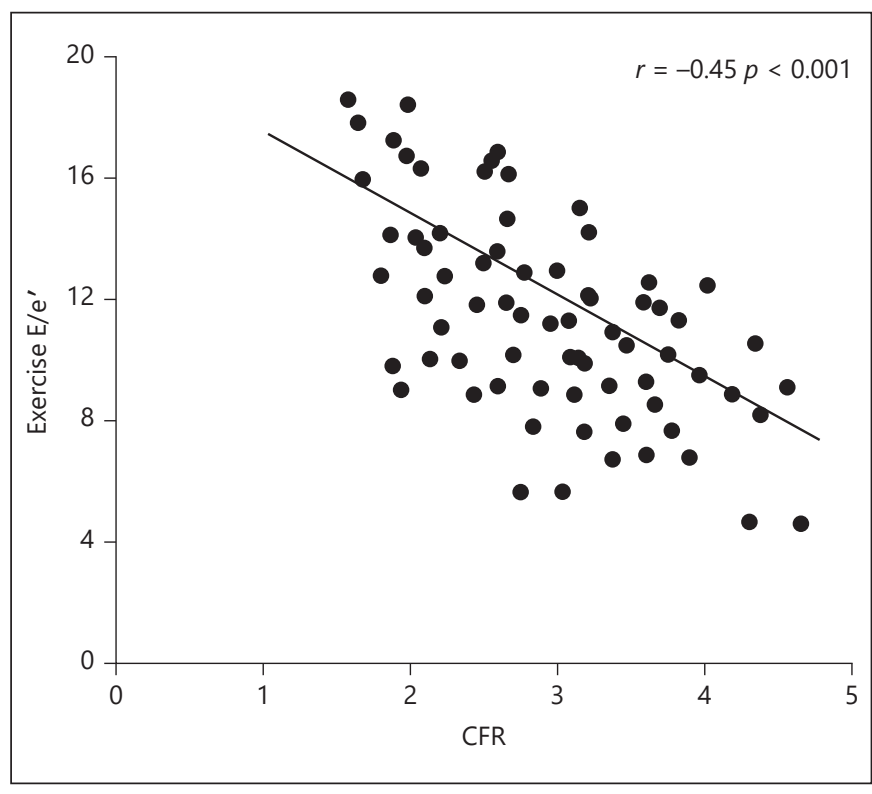

Fig. 2. Correlation between exercise E/e' and coronary flow reserve (CFR) in newly diagnosed hypertensives.

of symptoms (dyspnea and effort intolerance), normal ejection fraction, and echocardiographic evidence of diastolic dysfunction. Nevertheless, heart failure with preserved ejection fraction symptoms frequently occur during exercise, where LV filling pressure is usually normal at rest and only increases considerably during exercise.

Laine et al. [18] had supposed that coronary MVD may occur in early stages of hypertension, even in the absence of LV hypertrophy. This MVD may result in myocardial ischemia and fibrotic changes [19]. Impaired CFR is mainly related to minimal coronary resistance that is independent of vascular tone [20].

In hypertensive subjects, there is chronic pressure overload, which results in ultrastructural myocardial remodeling. Moreover, coronary MVD is usually developed early in those patients. These changes had been suggested to play a significant role in the incident heart failure with preserved ejection fraction $[21,22]$.

Furthermore, previous studies have shown that, even in the absence of LV hypertrophy, hypertensive patients have an impaired myocardial metabolism, which worsens during pharmacological stress testing [23]. Therefore, we believe that in the early stages of hypertensive heart disease, these intrinsic myocardial alterations, including microvascular coronary dysfunction and impaired myocardial metabolism, could explain the development of abnormalities of $\mathrm{LV}$ diastolic function that are evident only 
Table 5. Univariate and multivariate analysis of the variables associated with coronary flow reserve

\begin{tabular}{|c|c|c|c|c|c|c|}
\hline & \multicolumn{3}{|c|}{ Univariate } & \multicolumn{3}{|c|}{ Multivariate } \\
\hline & OR & $95 \% \mathrm{CI}$ & $p$ value & OR & $95 \%$ CI & $p$ value \\
\hline hs-CRP & 0.85 & $0.62-0.96$ & $<0.05$ & 0.30 & $0.15-0.43$ & 0.24 \\
\hline HR exercise & 0.65 & $0.48-0.91$ & 0.06 & & & \\
\hline LAVI & 0.75 & $0.49-1.06$ & $<0.05$ & 0.41 & $0.22-0.73$ & 0.15 \\
\hline LVMI & 0.62 & $0.59-0.80$ & $<0.08$ & & & \\
\hline Resting E/e' & 0.67 & $0.52-0.90$ & $<0.06$ & 0.35 & $0.13-0.65$ & 0.09 \\
\hline Exercise E/A & 0.65 & $0.48-0.91$ & $<0.06$ & & & \\
\hline Exercise E/e' & 1.53 & $1.17-2.11$ & $<0.001$ & 1.24 & $0.83-1.65$ & $<0.001$ \\
\hline
\end{tabular}

OR, odds ratio; CI, confidence interval; E/e', early mitral flow velocity to early mitral annular velocity; E/A, early mitral flow velocity to late mitral flow velocity; hs-CRP, high-sensitivity C-reactive protein; HR, heart rate; LAVI, left atrial wall index; LVMI, left ventricular mass index.

during exercise, when myocardial oxygen demand is greatest.

Mechanisms underlying the relationship of subclinical atherosclerosis and diastolic function are probably related to vascular function.

The association between subclinical atherosclerosis and impaired diastolic function is likely due to vascular functional alteration. Sonoda et al. [24] reported that CFR is related to increased vascular intima CFR. The increased microvascular intima is associated with impaired vasodilator reserve of the coronary microvasculature as a consequence of endothelial dysfunction, even in the absence of obstructive coronary artery disease. Moreover, Yambe et al. [25] concluded that atherosclerosis may augment aortic and carotid pressure, resulting in higher LV afterload and LV diastolic function. In fact, increased arterial stiffness is significantly associated with both atherosclerosis and impaired diastolic function.

\section{Limitations}

Several limitations were encountered. First, invasive assessment of LV filling was not performed. Accordingly, the exact rise of LV filling pressure cannot be obtained. Nevertheless, some investigators have described a significant correlation between invasive and noninvasive LV filling pressure assessment for subjects with normal and impaired diastolic stress echocardiography [11, 12]. Second, we used treadmill exercise to assess diastolic stress echocardiography. Third, invasive coronary angiography was not performed, therefore, we cannot exclude those with coronary stenosis for certain.

Diastolic Stress Echo, Hypertension

\section{Conclusion}

We have demonstrated significant associations between exercise-derived raised LV pressure and coronary MVD in newly diagnosed untreated hypertensive patients. Herein, we supposed that exercise-derived-E/e' could predict subclinical atherosclerosis and might explain the development of heart failure with preserved ejection fraction in hypertensive patients.

\section{Statement of Ethics}

The study was conducted ethically in accordance with the World Medical Association Declaration of Helsinki. All participants have given their written informed consent.

\section{Conflict of Interest Statement}

The authors have no conflicts of interest to declare.

\section{Funding Sources}

There are no funding sources to declare.

\section{Author Contributions}

R.A.M.: idea and design of the work, writing and submission of the paper as a corresponding author. M.A.: analysis and interpretation of data and share in writing the paper. I.G.: collection of data, analysis of data, writing and drafting the paper and revising it critically. M.E.: collection of data, writing and drafting the paper and revising it critically. 


\section{References}

1 Antony I, Nitenberg A, Foult JM, Aptecar E. Coronary vasodilator reserve in untreated and treated hypertensive patients with and without left ventricular hypertrophy. J Am Coll Cardiol. 1993 Aug;22(2):514-20.

2 Galderisi M, de Simone G, Cicala S, De Simone L, D’Errico A, Caso P, et al. Coronary flow reserve in hypertensive patients with appropriate or inappropriate left ventricular mass. J Hypertens. 2003 Nov;21(11):2183-8.

3 Brush JE Jr, Cannon RO 3rd, Schenke WH, Bonow RO, Leon MB, Maron BJ, et al. Angina due to coronary microvascular disease in hypertensive patients without left ventricular hypertrophy. N Engl J Med. 1988 Nov; 319(20):1302-7.

4 Nagueh SF, Smiseth OA, Appleton CP, Byrd BF 3rd, Dokainish H, Edvardsen T, et al. Recommendations for the evaluation of left ventricular diastolic function by echocardiography: an update from the American Society of Echocardiography and the European Association of Cardiovascular Imaging. J Am Soc Echocardiogr. 2016 Apr;29(4):277-314.

5 Nagueh SF, Smiseth OA, Appleton CP, Byrd BF 3rd, Dokainish H, Edvardsen T, et al.; Houston, Texas; Oslo, Norway; Phoenix, Arizona; Nashville, Tennessee; Hamilton, Ontario, Canada; Uppsala, Sweden; Ghent and Liège, Belgium; Cleveland, Ohio; Novara, Italy; Rochester, Minnesota; Bucharest, Romania; and St. Louis, Missouri. Recommendations for the evaluation of left ventricular diastolic function by echocardiography: an update from the American Society of Echocardiography and the European Association of Cardiovascular Imaging. Eur Heart J Cardiovasc Imaging. 2016 Dec;17(12):1321-60.

6 Lancellotti P, Pellikka PA, Budts W, Chaudhry FA, Donal E, Dulgheru R, et al. The clinical use of stress echocardiography in non-ischaemic heart disease: recommendations from the European Association of Cardiovascular Imaging and the American Society of Echocardiography. J Am Soc Echocardiogr. 2017 Feb;30(2):101-38.

7 D'Andrea A, Nistri S, Castaldo F, Galderisi M, Mele D, Agricola E, et al.; Working Group Nucleus on Echocardiography of Italian Society of Cardiology. The relationship between early left ventricular myocardial alterations and reduced coronary flow reserve in noninsulin-dependent diabetic patients with microvascular angina. Int J Cardiol. 2012 Feb; 154(3):250-5.
8 Nelson MD, Szczepaniak LS, Wei J, Haftabaradaren A, Bharadwaj M, Sharif B, et al. Diastolic dysfunction in women with signs and symptoms of ischemia in the absence of obstructive coronary artery disease: a hypothesis-generating study. Circ Cardiovasc Imaging. 2014 May;7(3):510-6.

9 Nagueh SF, Appleton CP, Gillebert TC, Marino $\mathrm{PN}$, Oh JK, Smiseth OA, et al. Recommendations for the evaluation of left ventricular diastolic function by echocardiography. J Am Soc Echocardiogr. 2009 Feb;22(2):10733.

10 Takagi T, Yoshikawa J. Diastolic stress echocardiography in Japanese elderly patients: prevalence and features of patients with elevated left ventricular filling pressure after treadmill stress. J Echocardiogr. 2011 Mar; 9(1):17-23.

11 Burgess MI, Jenkins C, Sharman JE, Marwick TH. Diastolic stress echocardiography: hemodynamic validation and clinical significance of estimation of ventricular filling pressure with exercise. J Am Coll Cardiol. 2006 May;47(9):1891-900.

12 Talreja DR, Nishimura RA, Oh JK. Estimation of left ventricular filling pressure with exercise by Doppler echocardiography in patients with normal systolic function: a simultaneous echocardiographic-cardiac catheterization study. J Am Soc Echocardiogr. 2007 May;20(5):477-9.

13 Ikonomidis I, Lekakis J, Papadopoulos C, Triantafyllidi H, Paraskevaidis I, Georgoula G, et al. Incremental value of pulse wave velocity in the determination of coronary microcirculatory dysfunction in never-treated patients with essential hypertension. Am J Hypertens. 2008 Jul;21(7):806-13.

14 Voci P, Pizzuto F, Mariano E, Puddu PE, Chiavari PA, Romeo F. Measurement of coronary flow reserve in the anterior and posterior descending coronary arteries by transthoracic Doppler ultrasound. Am J Cardiol. 2002 Nov; 90(9):988-91.

15 Holland DJ, Prasad SB, Marwick TH. Contribution of exercise echocardiography to the diagnosis of heart failure with preserved ejection fraction (HFpEF). Heart. 2010 Jul;96(13): 1024-8.

16 Holland DJ, Prasad SB, Marwick TH. Prognostic implications of left ventricular filling pressure with exercise. Circ Cardiovasc Imaging. 2010 Mar;3(2):149-56.
17 Ponikowski P, Voors AA, Anker SD, Bueno H, Cleland JG, Coats AJ, et al.; Authors/Task Force Members; Document Reviewers. 2016 ESC Guidelines for the diagnosis and treatment of acute and chronic heart failure: the Task Force for the diagnosis and treatment of acute and chronic heart failure of the European Society of Cardiology (ESC). Developed with the special contribution of the Heart Failure Association (HFA) of the ESC. Eur J Heart Fail. 2016 Aug; 18(8):891-975.

18 Laine H, Raitakari OT, Niinikoski H, Pitkänen OP, Iida H, Viikari J, et al. Early impairment of coronary flow reserve in young men with borderline hypertension. J Am Coll Cardiol. 1998 Jul;32(1):147-53.

19 Schwartzkopff B, Motz W, Frenzel H, Vogt M, Knauer S, Strauer BE. Structural and functional alterations of the intramyocardial coronary arterioles in patients with arterial hypertension. Circulation. 1993 Sep;88(3):9931003.

20 Frohlich ED. Fibrosis and ischemia: the real risks in hypertensive heart disease. Am J Hypertens. 2001 Jun;14(6 Pt 2):194S-9S.

21 Di Bello V, Pedrinelli R, Giorgi D, Bertini A, Talini E, Mengozzi G, et al. Coronary microcirculation in essential hypertension: a quantitative myocardial contrast echocardiographic approach. Eur J Echocardiogr. 2002 Jun;3(2):117-27.

22 Palombo C, Kozakova M, Magagna A, Bigalli G, Morizzo C, Ghiadoni L, et al. Early impairment of coronary flow reserve and increase in minimum coronary resistance in borderline hypertensive patients. J Hypertens. 2000 Apr; 18(4):453-9.

23 Schwartzkopff B, Brehm M, Mundhenke M, Strauer BE. Repair of coronary arterioles after treatment with perindopril in hypertensive heart disease. Hypertension. 2000 Aug;36(2): 220-5.

24 Sonoda M, Yonekura K, Yokoyama I, Takenaka K, Nagai R, Aoyagi T. Common carotid intima-media thickness is correlated with myocardial flow reserve in patients with coronary artery disease: a useful non-invasive indicator of coronary atherosclerosis. Int J Cardiol. 2004 Feb;93(2-3):131-6.

25 Yambe M, Tomiyama H, Hirayama Y, Gulniza Z, Takata Y, Koji Y, et al. Arterial stiffening as a possible risk factor for both atherosclerosis and diastolic heart failure. Hypertens Res. 2004 Sep;27(9):625-31. 